\title{
A NIR spectrum of a hot Jupiter from the ground: Preliminary results
}

\author{
Avi M. Mandell ${ }^{1}$, L. Drake Deming ${ }^{1}$, Geoffrey A. Blake ${ }^{2}$, Heather A. \\ Knutson $^{3}$, Michael J. Mumma ${ }^{1}$, Geronimo L. Villanueva ${ }^{1,4}$ \\ and Colette Salyk ${ }^{5}$ \\ ${ }^{1}$ NASA GSFC, ${ }^{2}$ Caltech, ${ }^{3}$ UC Berkeley, ${ }^{4}$ Catholic University, ${ }^{5}$ UT Austin \\ email: avi.mandell@nasa.gov
}

\begin{abstract}
High resolution NIR spectroscopy offers an excellent complement to the expanding dataset of transit and secondary eclipse observations of exo-planets with Spitzer that have provided the bulk of our understanding of the atmospheres and internal structure of these objects. High-resolution data can quantify the vertical temperature structure by isolating specific spectral lines formed at various depths. The presence of an opaque absorbing layer can also be inferred - and its pressure level determined quantitatively - via its effect on spectral line intensities.

We have analyzed data for a single secondary eclipse of the bright transiting exo-planet host star HD189733 at L-band wavelengths $(3-4 \mu \mathrm{m})$ using the NIRSPEC instrument on KeckII. We utilize a sophisticated first-order telluric absorption modeling technique that, combined with a calibration star, has already been proven to remove the effects of varying atmospheric transmittance and allow us to reach unprecedented $\mathrm{S} / \mathrm{N}$. We are conducting validation of the final data reduction products and developing high-resolution atmospheric models for comparison, but we have already been able to rule out emission from methane as reported by Swain et al. (2010). We present preliminary results and discuss future plans for analysis and observations.
\end{abstract}

Keywords. infrared: planetary systems, radiative transfer, techniques: spectroscopic

\section{Introduction}

Ground-based observations are now pushing the boundaries of our knowledge of exoplanet atmospheres. Near-infrared photometry of the hottest exoplanets is now possible using ground-based observatories (Gillon et al. 2009; Alonso et al. 2010; de Mooij \& Snellen 2009), and measurement of atmospheric absorption in strong atomic lines has also been successful from the ground (Redfield et al. 2008; Snellen et al. 2008). However, ground-based spectroscopic analysis of exoplanet atmospheres is in its infancy. Even at infrared wavelengths, short-period exoplanets are very dim compared with their parent star (at $5 \mu \mathrm{m}$ the planet-star contrast is $0.002-0.003$, while at $1 \mu \mathrm{m}$ the contrast drops to 0.0005 ), and many previous attempts to reach these levels of precision have been frustrated by the fundamental difficulties of observing in the infrared: emission and absorption by our own atmosphere. There have been several recent announcements of breakthroughs in ground-based spectroscopy: Snellen et al. (2010) announced a detection of wind motions in the atmosphere of HD209458 using high-resolution data of CO at $2 \mu \mathrm{m}$ from the VLT/CRIRES spectrograph, and a startling ground-based detection of a possible bright emission feature at $3.5 \mu \mathrm{m}$ from the atmosphere of the exoplanet HD 189733b was announced by Swain et al. (2010). While these early detections may eventually prove to be spurious, they emphasize the impact that ground-based spectroscopy will have on the field of exoplanet characterization. 
With a new generation of extremely large ground-based telescopes now being planned (Hook 2009), achieving successful ground-based spectroscopy of molecular features in exoplanet atmospheres becomes a major priority. Ground-based facilities not only provide a huge increase in collecting power and available observing time, but the resolving power of available instruments covers both low-resolution broad-spectrum instruments as well as high-resolution echelle spectrometers. Increasing spectral resolving power provides increasing diagnostic power with respect to both the chemical and thermal structure of the atmosphere. If ground-based capabilities can be successfully utilized for exoplanet spectroscopy, they can quickly revolutionize our understanding of the atmospheric structure of known exoplanets and eventually prepare us to explore the complex atmospheres of rocky super-Earths in the Habitable Zone.

\section{Towards A Measurement of The Day-Side Spectrum for HD 189733b at High Resolution}

In this article we describe a new strategy for removing telluric contamination and instrumental systematics to a very high degree, which has enabled us to push to the precision required to measure an exoplanet spectrum $(\mathrm{S} / \mathrm{N}>1000)$ for observations over the full range of near-IR wavelengths $(1-5 \mu \mathrm{m})$. We have developed custom data reduction algorithms that enable us to achieve fractional accuracy of less than a part in a thousand at wavelengths with significant telluric absorption at high resolution (see Figure 1). These analysis techniques were previously used to detect new molecular emission features from warm gas in circumstellar disks (Mandell et al. 2008), reaching line-continuum contrast sensitivities of better than 1/2000 at L-band wavelengths. To correct for changing atmospheric conditions and airmass, we utilize terrestrial spectral transmittance models synthesized with the LBLRTM atmospheric modeling code (Clough et al. 2005) based on line parameters from the HITRAN 2008 molecular database updated with the latest spectroscopic parameters (Rothman et al. 2009). We fit models for every AB image set, enabling us to compensate for changes over the night and between stellar targets, and we perform the same telluric removal routine on both the science target and the calibration star. The post-telluric removal residuals of the science and calibration stars are then differenced to remove remnant fringes due to internal reflections in the detector and other instrumental artifacts, as well as minor errors in the telluric model such as improper pressure broadening and isotopic ratios.

Stellar absorption features are removed by creating a stellar template from the data, using either a transit/eclipse method or averaging spectra that cover a wide range of radial velocity changes in the planet spectrum due to the rapid motion of hot exoplanets around their parent star. We then subtract this stellar template from each $\mathrm{AB}$ set in the data after shifting the template to the correct planetary velocity shift, leaving final residuals for each $\mathrm{AB}$ set with only the signal from the planet. We then combine all the residuals, shifting all the residual spectra to compensate for the planet's orbital motion. This relies on an accurate ephemeris for the orbit of the planet; however, this requirement can also allow us to solve for the planetary velocity exactly by fitting a model to the data using different orbital solutions. This process achieves results corresponding to an rms noise only slightly larger (a factor of 1.5 ) than that expected from the photon statistics.

The first exoplanet we have targeted is HD 189733b, due to its close proximity to the Sun (19 parsecs) and the favorable planet-star contrast expected based on Spitzer observations. We observed the star on July 13, 2009 with NIRSPEC on Keck at a resolving power of $\mathrm{R}=25,000$, acquiring a total of 200 images before, during, and after the secondary eclipse. HD 189733 is a main-sequence K-type star, and the $1.1 \mathrm{M}_{J}$ planet 

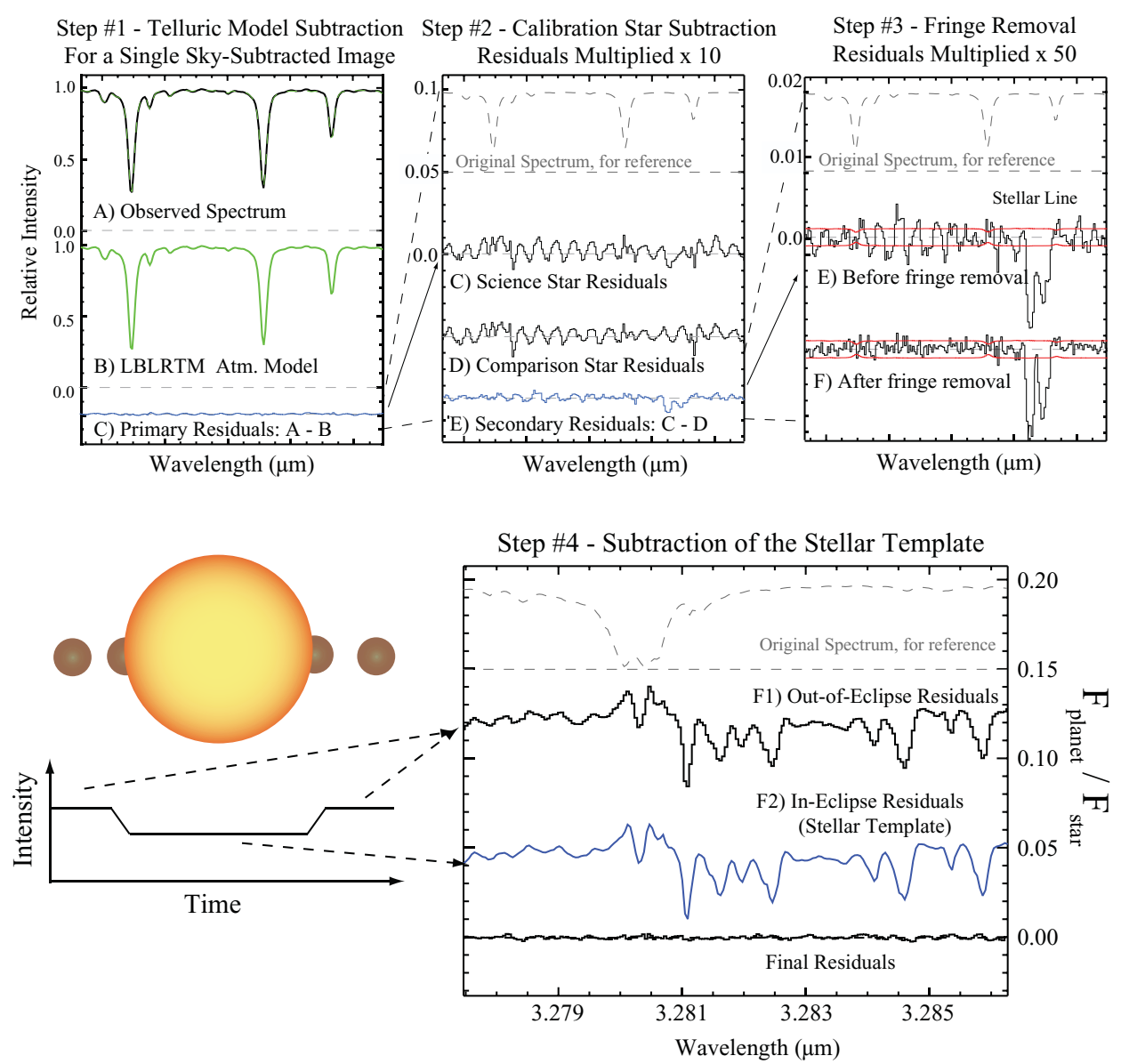

Figure 1. Demonstration of our data analysis procedure. In Step 1, synthetic terrestrial atmospheric models (B) are fitted separately to both the science and comparison stars (A). In Step 2, the two sets of residuals $(\mathrm{C}$ and $\mathrm{D})$ are then differenced to remove second-order instrumental or atmospheric features, leaving a secondary residual (E). In Step 3, remaining fringing is removed using a Fourier filter (F). This process is repeated for each AB set. In Step 4, stellar features are removed by subtracting the residuals from data during the eclipse of the planet (F2) from the combined residuals of the out-of-eclipse data (F1). The fractional accuracy on the stellar continuum in the final residuals is greater than 1 part in 1000 .

orbits in a circular orbit at 0.03 AU. We chose to observe a secondary eclipse of the planet at L-band wavelengths $(3-4 \mu \mathrm{m})$; our current wavelength coverage spans 4 spectral orders between $3.25 \mu \mathrm{m}$ and $3.8 \mu \mathrm{m}$, each covering $0.05 \mu \mathrm{m}$. For observations of the day-side of hot exoplanets, longer wavelengths provide the best planet-star contrast, and sky background levels are still relatively low at these wavelengths. The second important advantage of the L-band spectral region is the large number of strong transitions of molecular species such as $\mathrm{H}_{2} \mathrm{O}, \mathrm{CH}_{4}$, and other simple molecules present. We primarily target spectroscopic features of water because it is abundant in hot Jupiters, and has many hundreds of well-determined "hot" transitions (Barber et al. 2006) that we expect to be prominent in the exoplanet spectrum, but not excited in the telluric spectrum. The spectral coverage will also include methane near $3.3 \mu \mathrm{m}$, as well as additional trace species such as $\mathrm{OH}(3.28 \mu \mathrm{m})$ and $\mathrm{H}_{3}^{+}(3.67 \mu \mathrm{m})$, both of which are observed in Solar 
HD189733, NIRSPEC/Keck, Order 23, July 2009

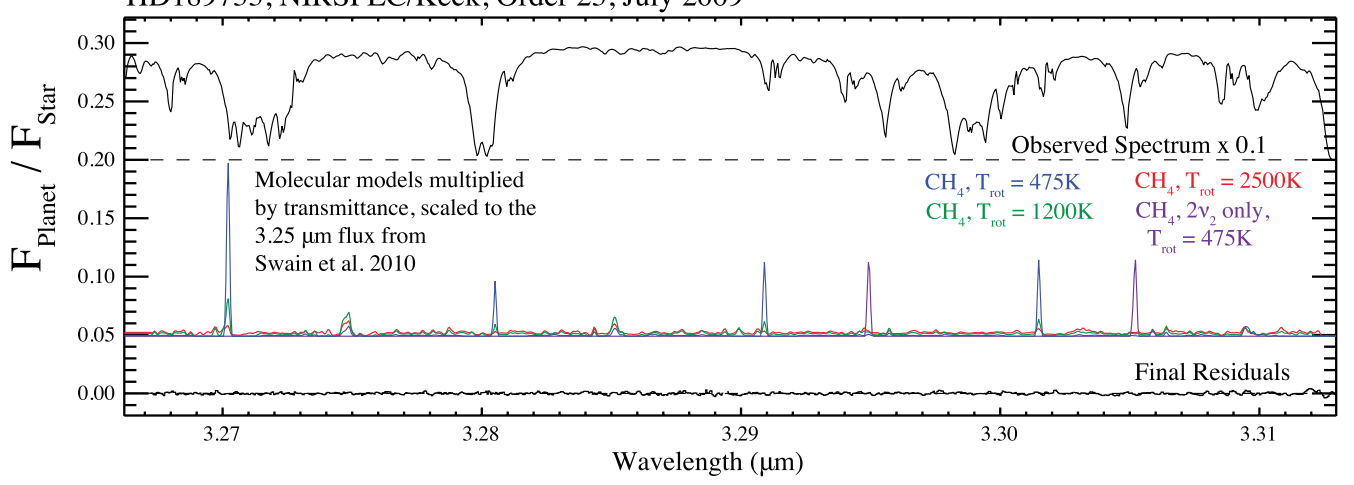

Figure 2. Results from our data reduction, with candidate molecular models for methane using the CH4@Titan line lists overplotted. The upper black trace is the original data, and the bottom black trace are our residuals after removing telluric and stellar features, shifting the data to the correct radial velocity and combining it, and then removing large-scale gradients in the continuum with a high-pass filter. We detect no emission at any of the expected positions, with upper limits more than 10 times below the total intensity of the expected emission for each model.

System objects and which may be excited by radiative pumping in the upper atmosphere of the exoplanet (cf. Shkolnik et al. (2006)).

\subsection{Non-Detection of Expected Bright L-Band Emission}

Our data has a theoretical contrast limit of 0.0005 , and our current analysis reaches an rms of 0.0011 . This is not sufficient to detect an unambiguous spectrum of the planet based on the expect line-to-continuum contrast from models, but our current level of precision allows us to easily search for evidence of the bright non-LTE emission from the exoplanet HD $189733 \mathrm{~b}$ at $3.25 \mu \mathrm{m}$ recently reported by Swain et al. (2010) based on observations at low spectral resolving power $(\lambda / \delta \lambda \approx 30)$. Non-LTE emission lines from gas in an exoplanet atmosphere will not be significantly broadened by collisions, so the measured emission intensity per resolution element must be substantially brighter when observed at high spectral resolving power, and we should be able to easily detect and characterize any planetary emission. We modeled the expected signal using ro-vibrational emission models of molecular species with transitions in the relevant wavelength region using a wide range of rotational excitation temperatures and vibrational level intensities, scaled to the results presented by Swain et al. (2010), in order to predict the line flux required at high resolution. We then compared it to our data; no flux was present at any of the expected transition frequencies between 3.27 and $3.31 \mu \mathrm{m}$, with upper limits 30 times smaller than the expected line fluxes, and similar limits were set for the other spectral orders we analyzed (Mandell et al. 2011).

The conditions that would lead to broad emission features beyond our detection limits are extremely difficult to reconcile with realistic models and previous observations. Additionally, our analysis indicates that the emission, if real, is too bright to be produced by fluoresence. Our wavelength region, even including all four spectral orders from our data, only covers a small section of the spectrum published by S10, and we cannot rule out an emission mechanism that produces flux outside our band passes. Additionally, we cannot rule out an exotic highly time-variable stellar emission process such as chargedparticle excitation due to flares. However, we regard these explanations as improbable, 
and conclude that inadequate telluric correction is the most likely explanation for the Swain et al. (2010) results.

\section{Conclusion}

Ground-based exoplanet spectroscopy has only recently born fruit, but holds great promise in our quest to investigate exoplanet atmospheres. Low-resolution spectroscopy over a wide spectral range $(1-5 \mu \mathrm{m})$ with moderate-resolution instruments provides constraints on the thermal continuum and overall molecular band shapes, while utilizing high-resolution spectroscopy over narrower spectral ranges allows us to measure individual molecular line strengths and shapes and constrain the temperature at which the molecular absorption features are formed. Detection of specific molecular features in an exoplanet spectrum will allow us to quantify temperature versus height by isolating combinations of lines formed over a range of height and comparing their intensities. These analysis techniques will allow us to probe the chemical and thermal structure of exoplanet atmospheres by measuring the strengths and line shapes for individual molecular transitions of important molecular constituents such as $\mathrm{H}_{2} \mathrm{O}, \mathrm{CH}_{4}$, and $\mathrm{CO}$ at moderate and high resolution, removing degeneracies in the fitting of molecular bands and directly probing the vertical temperature structure of the atmosphere and allowing us to potentially resolve much of the current ambiguity in the modeling of exoplanet atmospheres (Madhusudhan \& Seager 2009). Longer wavelengths $(3-5 \mu \mathrm{m})$ can provide much better sensitivity for secondary eclipse measurements (which measures the thermal emission from the day side of the planet), while shorter wavelengths favor transit spectroscopy for planets with large atmospheric scale-heights. By combining multiple regimes in spectral resolving power and wavelength range, these observations hold the potential to revolutionize our ability to characterize the atmospheric structure of exoplanets in a variety of stellar and orbital configurations, and will help to prepare us for future observations with JWST.

\section{Acknowledgements}

A.M.M. was supported by the Goddard Center for Astrobiology and the NASA Postdoctoral Fellowship Program. H.A.K. is supported by a fellowship from the Miller Institute for Basic Research in Science.

\section{References}

Alonso, R., Deeg, H. J., Kabath, P., \& Rabus, M. 2010, AJ, 139, 1481

Barber, R. J., Tennyson, J., Harris, G. J., \& Tolchenov, R. N. 2006, MNRAS, 368, 1087

Clough, S. A., Shephard, M. W., Mlawer, E. J., et al. 2005, JQSRT, 91, 233

de Mooij, E. J. W. \& Snellen, I. A. G. 2009, A\&A, 493, L35

Gillon, M., Demory, B.-O., Triaud, A. H. M. J., et al. 2009, A\&A, 506, 359

Hook, I. 2009, in: Science with the VLT in the ELT Era, 225

Madhusudhan, N. \& Seager, S. 2009, ApJ, 707, 24

Mandell, A. M., Mumma, M. J., Blake, G. A., et al. 2008, ApJ, 681, L25

Mandell, A. M., Deming, L. D., Blake, G. A., et al. 2011, ApJ, 728, id.18

Redfield, S., Endl, M., Cochran, W. D., \& Koesterke, L. 2008, ApJ, 673, L87

Rothman, L. S., Gordon, I. E., Barbe, A., et al. 2009, JQSRT, 110, 533

Shkolnik, E., Gaidos, E., \& Moskovitz, N. 2006, AJ, 132, 1267

Snellen, I. A. G., Albrecht, S., de Mooij, E. J. W., \& Poole, R. S. L. 2008, A\&̊A, 487, 357

Snellen, I. A. G., de Kok, R. J., de Mooij, E. J. W., \& Albrecht, S. 2010, Nature, 465, 1049

Swain, M. R., Deroo, P., Griffith, et al. 2010, Nature, 463, 637 